\title{
A Study on the Cytotoxicity of Carbon-based Materials
}

\section{Dipendu Saha1, ${ }^{\text {, }}$, Caryn L. Heldt ${ }^{2}$, Maria F. Gencoglu², K. Saagar Vijayaragavan², Jihua Chen ${ }^{3}$, Ashish Saksule²}

1. Department of Chemical Engineering, Widener University One University Place, Chester, PA 19013, USA,

2. Department of Chemical Engineering, Michigan Technological University,1400 Townsend Dr., Houghton, MI, 49931, USA,

3. Center for Nanophase Materials Sciences, Oak Ridge National Laboratory. Oak Ridge, TN 37831, USA

*Corresponding author's E-mail: dsaha@mail.widener.edu (D. Saha), Phone: +1 610499 4056, Fax: 6104994059

To be submitted to Materials Science and Engineering: $C$ 


\begin{abstract}
With an aim to understand the origin and key contributing factors towards carboninduced cytotoxicity, we have studied five different carbon samples with diverse surface area, pore width, shape and size, conductivity and surface functionality. All the carbon materials were characterized with surface area and pore size distribution, x-ray photoelectron spectroscopy (XPS) and electron microscopic imaging. We performed cytotoxicity study in Caco-2 cells by colorimetric assay, oxidative stress analysis by reactive oxygen species (ROS) detection, cellular metabolic activity measurement by adenosine triphosphate (ATP) depletion and visualization of cellular internalization by TEM imaging. The carbon materials demonstrated a varying degree of cytotoxicity in contact with Caco-2 cells. The lowest cell survival rate was observed for nanographene, which possessed the minimal size amongst all the carbon samples under study. None of the carbons induced oxidative stress to the cells as indicated by the ROS generation results. Cellular metabolic activity study revealed that the carbon materials caused ATP depletion in cells and nanographene caused the highest depletion. Visual observation by TEM imaging indicated the cellular internalization of nanographene. This study confirmed that the size is the key cause of carbon-induced cytotoxicity and it is probably caused by the ATP depletion within the cell.
\end{abstract}




\section{Introduction}

Among carbon-based materials, $\mathrm{sp}^{2}$ hybridized carbons are the most common forms and largest fraction of carbon materials fall into this category. In $\mathrm{sp}^{2}$ carbons, the skeletons are formed by the alternate hexagonal single and double bonds between the carbons atoms and graphite is the ideal crystalline $\mathrm{sp}^{2}$ carbon material. The key skeletal structure of graphite-like $\mathrm{sp}^{2}$ materials remains the same, however, with more chemical and structural alternations of their morphology. The examples of such carbons include nano-carbons, like carbon nanotube or graphene, porous carbon, like activated microporous or mesoporous carbons or non-porous carbons, like carbon soot or carbon fiber [1,2]. In today's perspective, a very large number of $\mathrm{sp}^{2}$ carbon-based materials are employed in biological and biomedical platforms, including drug delivery, artificial implants, imaging agents or poison arresters. Like any other foreign materials, biocompatibility of these materials always remains questionable and past results obtained so far often contain conflicting or counter-intuitive results and explanations that prohibit any generalized conclusion.

Carbon materials have been shown to have toxic effects. The toxicity effects could possibly arise from the shape and size, surface functionality, hydrophilicity, porosity, surface conductivity or often the toxic guest species that are associated with these materials during processing. Carbon nanotubes (CNTs) are one of the early $\mathrm{sp}^{2}$ carbon materials that were studied for possible toxicity and since then, the popular term 'nanotoxicity' has evolved. Albeit conflicting results have been obtained on CNT based toxicity, it has almost been universally accepted that CNTs are toxic [1,3-7] and their toxicity is a strong function of agglomeration, functionalization and metallic catalyst contents [8-10]. It was demonstrated that sulfonic acid and meta-phthalic acid functionalized CNTs reveal much reduced cytotoxicity compared to non-functionalized CNTs and hydrophilicity was identified as the key factor [11]. Recently, Shi et al. [12] provided the visual evidence that the asymmetric shape of CNTs causes cell penetration. Graphene is an $\mathrm{sp}^{2}$ and 2D carbon nanomaterial that has already developed its potential in biomedical platforms. The malicious effect of graphene has already been demonstrated from cellular to ecological studies. Researchers demonstrated that the sharp edge of 
graphene penetrates through the cellular membrane [13] and apparently the 'acicular' shape of the object causes cell penetration and possible toxicity. Riding et al. [14] demonstrated the mechanistic understanding of nanocarbon toxicity in biochemical level on both pro- and eukaryotic cells with the help of multi-beam synchrotron radiation-based Fourier-transform infrared imaging (SR-FTIRI) at diffraction-limited resolution. Similar to CNTs, functionalization of graphene with hydrophilic entities also proved to reduce its cytotoxic behavior [15]. Likely, hydrophilicity causes the nanomaterials to be well dispersed in water and statistically lowers the direct cell-material interactions, thus reducing deposition of the materials onto the cell membrane. Additionally, hydrophilic surfaces interact with proteins through water molecules, thereby minimizing protein degradation [16]. On the contrary, a hydrophobic surface directly interacts with cells, causing conformational changes and denaturation of protein, [16] thereby resulting in diverse cellular malfunctions [17].

Other than nanocarbons materials, bulk or monolithic carbon materials are also used in biomedical applications. Oral delivery of activated carbon suspensions has long been used as a poison arrester for accidental toxin ingestion or drug overdose [18,19]. Recently, there is a significant growth in the application of mesoporous carbons in drug delivery for a diverse array of drugs; both bulk [2,20-26] and nano-sized [27-29] mesoporous carbons have been employed for these purposes. In our previous work, we have demonstrated that mesoporous carbon material was non-toxic and its benign nature was confirmed through cell viability, protein adsorption and hemolysis studies [30]. Although cytotoxicity studies did not reveal significant toxicity to HeLa cells, higher surface area carbons were found to be slightly more toxic compared to lower surface area carbons. While employing porous carbons or even porous silica materials in biological platforms, different parameters also appear to play significant role in causing toxicity to cells, like surface area [31-33] surface functionality [33] (likely due to the surface hydrophilicity/hydrophobicity) or even the conductivity related to electron transfer mechanisms [33]. 
Considering all the apparent contributing factors towards toxicity and with greater implementation of carbon materials in biological and biomedical platforms, there is a need for a generalized toxicity study of different varieties of carbon materials with proper controls. Additionally, it has been demonstrated that two distinct phenomena cause cytotoxicity, oxidative stress of the cell causing reactive oxygen species (ROS) generation and reduction of cellular activity by adenosine triphosphate (ATP) depletion resulting in ultimate cell apoptosis or necrosis. For carbon-based materials, these two phenomena were not investigated in detail, and to the best of our knowledge, the exact root of cellular toxicity of carbons materials is not known. In this phase of work, we have selected five types of carbon materials with variation in size and shape, surface area, surface functionality and conductivity. The carbon materials that we selected for this study are non-porous carbon (C1), highly porous commercial carbon with very high surface area (C2), mesoporous carbon with low surface area and large pore width (C3), graphite powder with highest conductivity (C4) and nanographene with moderate surface area and high conductivity (C5) as a representative of nano-carbon. We did not include carbon nanotube (CNT) in our study as it was already studied several times in the past and several additional control parameters associated with CNTs would make the conclusion of study more complex. We employed Caco-2 (human colon carcinoma cell line) as a model cell to study the cytotoxicity as this type of cell line is regarded as a model cell for intestinal epithelia cells [34]. All the materials were characterized by porosity analysis, surface functionality and conductivity by x-ray photoelectron spectroscopy (XPS) and particle size and shape determination by scanning electron microscopy (SEM) and transmission electron microscopy (TEM). We have examined the cytotoxicity study by a colorimetric assay, ATP depletion by a luminescent assay, reactive oxygen species (ROS) generation by a fluorescence assay, and visual inspection of cell internalization by light and electron microscopy.

\section{Materials and methods}

\subsection{Carbon Materials and Characterization}

Among the materials studied in this phase of work, non-porous carbon (C1) and mesoporous carbon (C3) were synthesized in the laboratory. The key principle of synthesis 
of $\mathrm{C} 1$ is very similar to that reported previously in the literature [35] without the activation step. Typically, $10 \mathrm{~mL}$ of furfuryl alcohol is polymerized by adding $5 \mathrm{~mL}$ of $0.1 \mathrm{M}$ toluenesulfonic acid dissolved in tetrahydrofuran as a catalyst. In order to avoid a vigorous reaction, the addition of the two reagents is controlled in a very slow fashion $(\sim 10 \mathrm{~mL} / \mathrm{h})$ and the reaction mixture is cooled in an ice bath. The reaction is continued for $48 \mathrm{~h}$ till it solidifies to a dark green, semi-solid mass. This is followed by carbonization in a tube furnace in a $\mathrm{N}_{2}$ atmosphere up to $1000^{\circ} \mathrm{C}$ and at a ramp rate of $10{ }^{\circ} \mathrm{C} / \mathrm{min}$ and subsequent cooling in the same $\mathrm{N}_{2}$ flow. Mesoporous carbon was also synthesized according to our previously published procedure [30]. Typically, $50 \mathrm{~g}$ resorcinol (carbon precursor) and 40 g F127 (template or structure dictating agent) were dissolved in a 20:1 volume ratio of water and ethanol was cross-linked with $48 \mathrm{~mL}$ of formaldehyde along with $60 \mathrm{~mL} 6 \mathrm{M} \mathrm{HCl}$ as a catalyst. Upon separating the polymer layer from the solvent, it was carbonized under a $\mathrm{N}_{2}$ flow in a porcelain boat in the specified conditions of $0{ }^{\circ} \mathrm{C}$ to $400{ }^{\circ} \mathrm{C}$ at a rate of 1 ${ }^{\circ} \mathrm{C} / \mathrm{min}$ and $400{ }^{\circ} \mathrm{C}$ to $1000{ }^{\circ} \mathrm{C}$ at a rate of $2{ }^{\circ} \mathrm{C} / \mathrm{min}$ and subsequent cooling in the same $\mathrm{N}_{2}$ flow. High surface area, commercially-available activated carbon (C2) and graphene (C5) were obtained from ACS Material and employed as received. Graphite powder was obtained from MTI Corporation and also employed as received.

The porosity, including BET surface area and pore size distributions of all the materials obtained was analyzed by the standard $\mathrm{N}_{2}$ adsorption-desorption at $77 \mathrm{~K}$ in Quantachrome's Autosorb iQ instrument. Scanning electron microscopic (SEM) images were obtained in an Evex Mini-SEM II HR-3000 model. The TEM images were obtained in a Carl Zeiss Libra 120 TEM operating at $120 \mathrm{kV}$. X-ray photoelectron spectroscopy (XPS) data was obtained in a Thermo-Fisher K-alpha instrument XPS system operating at monochromatic Al K- $\alpha$ as a X-ray anode. The x-ray energy employed was $1486.6 \mathrm{eV}$ and resolution of $0.5 \mathrm{eV}$.

\subsection{Toxicity Studies}

All toxicity studies were undertaken with Caco-2 cells (ATCC@ HTB-37). Caco-2 is a human epithelial cell line. The cells were grown at $37^{\circ} \mathrm{C}, 5 \% \mathrm{CO}_{2}$, and $100 \%$ humidity in DMEM (Life Technologies, Grand Island, NY) media that was supplemented with $10 \%$ fetal 
bovine serum (Atlantic Biologicals, Atlanta, GA) and 1\% penicillin and streptomycin (Life Technologies, Grand Island, NY). Cell toxicity was measured with an MTT Assay, as described earlier [30], except experiments were conducted with Caco-2 cells and not HeLa cells.

ATP was measured with the CellTiter-Glo® Luminescent Cell Viability Assay from Promega (Madison, WI). Caco-2 cells were seeded at a density of $2 \times 10^{5}$ cells $/ \mathrm{ml}$ with 100 $\mu \mathrm{l}$ per well in a white 96-well plate and allowed to grow for 24 hours. Carbon materials were made in 100 and $300 \mu \mathrm{l} / \mathrm{ml}$ solutions in DMEM supplemented media and sonicated with a Heat Systems Ultrasonic XL sonicator (Plainville, NY) for 5 minutes. Each carbon was added to the wells in a volume of $25 \mu \mathrm{l}$. At different times, the cells were equilibrated to room temperature for 30 minutes $[36,37]$, followed by addition of the the CellTiterGlo ${ }^{\circledR}$ reagents, per manufacturer instructions. The chemiluminescence was read after a 10 minute incubation of the reagents [36,37] on a Thermo Fluoroskan Ascent FL (Grand Island, NY). 9\% Triton-X was used as a positive control and phosphate buffer saline (PBS) (Life Technologies, Grand Island, NY) was used as a negative control.

ROS was measured with CellRox® Green fluorogenic reagent (Thermo-Fisher, Grand Island, NY). Cells were treated the same as the ATP assay except the cells were seeded into a black 96-well plate. After equilibration to room temperature, the CellRox ${ }^{\circledR}$ reagents were added to the cells and carbon samples and incubated for 30 minutes at $37^{\circ} \mathrm{C}$. The media was removed and the cells were washed with PBS three times. The fluorescence (excitation: 485nm/emission: 520nm) was read on a Synergy Mx microplate reader (BioTek, Winooski, VT). Hydrogen peroxide at $0.25 \%$ and $0.5 \%$ was used as positive controls and PBS as a negative control.

\subsection{Cell Imaging}

Light microscopy images were prepared as described earlier for the ATP assay except the cells were seeded into a clear 96-well plate and images were obtained 24 hours 
after carbon addition. Images were obtained with an Olympus IX51 microscope equipped with a DP72 camera (Olympus, Center Valley, PA).

For TEM imaging, Caco-2 cells were grown to $90 \%$ confluency in 6-well plates. Carbon samples were sonicated at a concentration of $100 \mu \mathrm{g} / \mathrm{ml}$, as described earlier, in completed media. The sonicated samples were added to the cells in a 1:4 dilution of media already on the cells. This is the same concentration as the toxicity studies. After 4 hours, the media was removed. The cells were fixed with $2 \%$ glutaraldehyde (electromicroscopy grade, Sigma, St. Louis, MO) in $0.1 \mathrm{M}$ sodium cacodylate buffer at $\mathrm{pH} 7.4$ for 2 hours at room temperature. The cells were washed three times with the cacodylate buffer. They were then fixed a second time with $1 \%$ osmium tetroxide in the cacodylate buffer for 1 hour at $4^{\circ} \mathrm{C}$. This was followed by three washes with cacodylate buffer. The cells were scraped and placed into a microcentrifuge tube. The buffer was removed by centrifugation and removal of the supernatant. The cells were then enrobed in a 1.5\% agarose solution and cooled to $4^{\circ} \mathrm{C}$. Then they were dehydrated with subsequent changes in ethanol at $30,50,70,95$, and $100 \%$ ethanol. The dehydrated cells were infiltrated with propylene oxide in ethanol by subsequent changes in $35,50,75,95$, and $100 \%$ propylene oxide. The sample was mounted with a $1: 1$ propylene oxide/epoxy resin followed by a $75 \%$ epoxy resin/25\% propylene oxide and accelerator and finally a 100\% epoxy resin/accelerator composition. The cells were embedded into a BEAM capsule and cured at $60^{\circ} \mathrm{C}$ for 48 hours. The BEAM capsule was sliced, mounted on a copper grid, stained with uranyl acetate and lead citrate and allowed to dry. The final stained sections were examined with a JEOL JEM-2010.

\section{Results}

\subsection{Materials Characteristics}

Amongst the materials explored, C2 has the highest surface area (BET: $2194 \mathrm{~m}^{2} / \mathrm{g}$ ) in contrary to $\mathrm{C} 1$ and $\mathrm{C} 4$ that are practically non-porous. The BET surface areas of all of these materials are provided in table 1. The SEM/TEM images (figure 1) provided an estimation of particle size of all the carbon materials. The smallest particle size belonged to C5 and C2. The size of C5 (graphene) samples are less than a micron $(0.25 \mu \mathrm{m}$ to $50 \mathrm{~nm})$, whereas the C2 sample size was within 5 to $10 \mu \mathrm{m}$. Graphite particles have the 
intermediate size range of 20-30 $\mu \mathrm{m}$. The particle size of the mesoporous carbon samples (C3) are heterogeneous, the larger particles are in the range of $200 \mu \mathrm{m}$, whereas the smaller particles are within 20-60 $\mu \mathrm{m}$. The largest particle size was found for $\mathrm{C} 1$, which are 100 to over $500 \mu \mathrm{m}$.

The identification and quantification of surface functionality of these carbon materials are performed by XPS results. Owing to the daunting task of determining hydrophilicity/hydrophobicity by traditional contact angle measurement for powdered materials, it is mentioned that the surface oxygen and oxygen bearing functional group contents could provide an indirect measurement of hydrophilicity [38]. As the oxygen containing functional groups of $C-O, C=O, O-C=O$ or $C-O-H$ can form a hydrogen bond with water, a higher percentage of oxygen or these oxygen bearing functional groups may indicate a higher degree of hydrophilicity. Figure 2 shows the peak fitting results of the XPS analysis of the carbon materials and quantification data shown in table 1. Based on this assumption, C5 (graphene) possesses the highest degree of hydrophilicity followed by C4 and $\mathrm{C} 2$.

\subsection{Cytotoxicity}

The optical microscopy of the carbon particles in contact with Caco-2 cells and the cytotoxicity studies by the colorimetric MTT cell proliferation assay are shown in figure 3 and 4, respectively. These light microscopic images provide a preliminary understanding on the degree of dispersion of the carbon materials in the course of the experiment as well as an overall estimate on the size ratio of carbon to cells. They reveal that the carbon particles are in contact with Caco- 2 cells and well dispersed in the media. The cytotoxicity study was limited by the maximum carbon concentration of $300 \mu \mathrm{g} / \mathrm{ml}$ as the higher concentration of carbon interferes with the colorimetric assay. At higher concentration, the result demonstrates an apparent decrease in cytotoxicity (or increase in cell survival), which does not reflect the true results of the experiment. In the findings, it is observed that the C3 (mesoporous carbon) demonstrates the least cytotoxicity compared to the other carbon samples and the cell survival is over $95 \%$ at the highest carbon concentration. Slight increase in cellular survival to over $100 \%$ for this sample may be attributed to the 
additional surface provided by the large particles for the cells to grow [30]. Sample C1 (non-porous large carbon particle) demonstrates gradual decrease in cell survival with the increase in its concentration. All of the samples of C2, C4 and C5 revealed apparent decrease in cell survival rate with the increase in carbon concentration and C5 (nanographene) causes the most cytotoxic behavior compared to the rest of the samples. For C5, the minimal cell survival was below $70 \%$ at the concentration of $150 \mu \mathrm{g} / \mathrm{ml}$. The slight increase in cell survival for $\mathrm{C} 5$ at the highest carbon concentration may be attributed to the interference of graphene with the colorimetric assay. The cytotoxicity results suggested that except for $\mathrm{C} 1$, which has the largest particle size, higher cytotoxicity is manifested by the smaller particles and the smallest (nano) particle is proved to be the most cytotoxic for this cell line.

\subsection{Reactive oxygen species (ROS) generation}

Generation of reactive oxygen species (ROS) is the signature of oxidative stress to cells. Oxidative stress causes severe deleterious effects to cells, often leading to cell death. Figure 5(a) and (b) show the results of the ROS generation by all the carbon particles after 4, 8, 12 and 24 hours of incubation, as observed by analyzing the concentration of superoxide ions. Quite unexpectedly, none of the carbons demonstrated statistically significant ROS generation compared to the control (blank media), even after 24 hours of exposure. Therefore, this result confirms that the cytotoxic behavior of $\mathrm{C} 2, \mathrm{C} 4$ or $\mathrm{C} 5$ is not caused by oxidative stress to this cell line.

\subsection{ATP depletion}

The amount of adenosine triphosphate (ATP) in a cell culture is directly proportional to the metabolic activity of number of living cells. Lowering of ATP content probably induces mitochondrial disruption and cell death. The ATP depletion assays of these materials are shown in figure 6. The ATP contents were measured after 4 and 24 hours of exposure and the slight increase in ATP content is likely attributed to the amount of cell proliferation that likely occurred between 4 and 24 hours. C5 (nanographene) demonstrates the lowest ATP content of 7-20 \% in 4 to 24 hours of exposure followed by C2 (50-60\% remaining ATP content) as compared to the cells that were exposed only to the 
buffer. C4 (75-90\% remaining ATP content) and C3 (77\% remaining ATP content) revealed a small decrease in ATP content at the elevated carbon concentration of $300 \mu \mathrm{g} / \mathrm{ml}$. Although the cytotoxicity study by colorimetric assay showed that $\mathrm{C} 4$ decreases cellular survival almost similar to that of $\mathrm{C} 2$, it did not cause significant ATP depletion and the depletion rate is very similar to that of C3. C3 demonstrated the best cellular survival rate according to figure 4 . In contrary to cytotoxicity study, sample C1 did not cause any ATP depletion and so far, it showed the best ATP survival rate.

\subsection{Cellular Internalization}

In order to observe the cellular internalization of carbon particles, we employed C5 (nanographene, smallest particle size) as the representative sample to study under transmission electron microscopic (TEM) imaging. These TEM imaging results provided the direct evidence of cellular internalization of nanographene by Caco-2 cells. (figure 7 (a-c)). According to these three figures, the average size of nanographene within the cellular matter is 0.1 to $1 \mu \mathrm{m}$ and the majority of the particles are within the smaller range. Although not directly revealed in the images, it is quite possible that even smaller particles might have also entered the cytoplasmic matter. These particles are quite randomly dispersed within the cytoplasm but remained excluded from the vesicles. Although not directly measured in our study, the internalization of nanographene may not cause physical damage to plasma membrane as suggested by Sasidharan et al. [15] and toxicity induced by nanographene may directly be related to the ATP depletion as observed earlier.

\section{Discussion}

Cytotoxicity data confirmed that different types of carbon materials possess different responses in cytotoxicity tests for Caco-2 cells. So far, all the samples except C3 revealed different levels of cytotoxicity. $\mathrm{C} 5$ demonstrated to be most cytotoxic material. In two other following tests of ROS determination and ATP depletion, C1 did not reveal any harmful effect. Based on this comparison, it can be conferred that C1 is not cytotoxic and the apparent decrease in cellular activity with $\mathrm{C} 1$ in cytotoxicity study may be attributed to it's large size ( $\mathrm{C} 1$ is the largest amongst all the samples). Most likely, at the higher 
concentration, the bulky size of C1 provided a 'physical hindrance' to the growth of cells along with diminishing transfer of nutrient matter that resulted in lowering of cell survival.

Oxidative stress to the cells resulting in generation of reactive oxygen species (ROS) is a known cause of cytotoxicity and it has been proved that Caco-2 cells are prone to such damage $[39,40]$. Remarkably, none of the carbon samples with concentration as high as $300 \mu \mathrm{g} / \mathrm{ml}$ demonstrated statistically insignificant ROS generation based on the control. Although analyzed with different cells, our result contradicts the past results that reported significant ROS generation by graphene [15] or CNT [41] in macrophage or phagocytic cells. It was demonstrated that unfunctionalized graphene causes as high as $24.2 \%$ increase in ROS generation in the macrophage cells [15] in a concentration as low as $75 \mu \mathrm{g} / \mathrm{ml}$. Previous studies claimed that the surface electronic properties, like conductor or semiconductor behavior can facilitate the ROS formation or its transmission [33]. In our studies, graphite (C4) and nanographene (C5) were most conductive to electronic transfer, but they did not facilitate the ROS formation. It could be possible that ROS generation is cell specific. However, it needs to be mentioned that the carbon samples did not cause oxidative stress to Caco-2 cells, which are otherwise known to be susceptible to ROS damage [39-40] and it definitely provides a positive feature to the carbon samples compared to other synthetic substances that were employed as biomaterials. Oxidative stress causes several detrimental effects in cellular level that results in wide spectrum of cellular disorder. These disorders may manifest itself in terms of cell membrane peroxidation, few specific sicknesses, protein denaturation [42,43] and DNA strand breaking [42-44] causing carcinogenic effects. Past results demonstrated that mesoporous silica materials, like SBA15 or MCM-41 causes ROS generation in Caco-2 cells $[33,36]$ and it definitely provides an advantage of carbon-based materials over silica as novel biomaterials.

Unlike oxidative stress, the carbon samples demonstrated significant concentration dependent ATP depletion in at least two of the materials (C5 and C2) and partially in C3 and C4. Since these materials did not demonstrate statistically significant ROS generation, most likely, the root cause of cytotoxicity is attributed to the ATP depletion for Caco-2 cells. In earlier research efforts on silica materials, it was mentioned that an excess level of ROS 
could trigger to ATP depletion by mitochondrial disruption [36]. As significant ROS generation was not observed in our study, most likely, mitochondrial disruption could directly be caused by the carbon particles internalized by the cells themselves.

The key contributing factors that have been emphasized in the study of cytotoxicity are surface area, surface functionality including hydrophilicity/hydrophobicity and the size and shape. The two samples that demonstrated a significant cytotoxic behavior in ATP depletion study are C2, C5 and partially C3 and C4 Amongst these samples C5 demonstrated the highest toxicity. Within these materials, $\mathrm{C} 2$ has the highest BET surface area $\left(2194 \mathrm{~m}^{2} / \mathrm{g}\right)$, but C4 possesses the least BET surface area of around $2 \mathrm{~m}^{2} / \mathrm{g}$. C3 and C5 have the moderate surface areas of 315 and $514 \mathrm{~m}^{2} / \mathrm{g}$, respectively. Owing to lack of trend, it can be concluded that BET surface area has no specific influence in the cytotoxicity of these materials and most likely did not play any role for the carbon samples investigated in this study. Surface functionality could be another contributing factor as it may lead to hydrophilic or hydrophobic nature of the particles. According to XPS studies, C5 has the highest oxygen content leading to maximum oxygen functionality (13.2\%) in edge sites and thus possesses a higher degree of hydrophilicity compared to others. However, according to both colorimetric assay and ATP depletion studies, it demonstrated the highest toxicity compared to other samples. Previous studies confirmed that further functionalizing or coating graphene with hydrophilic polymers, like PEG or PEO inhibited its cytotoxic nature. Although surface functionality results demonstrated that the graphene possesses the relatively higher degree of apparent hydrophilicity compared to other samples, quite obviously, the present functionality was not enough improve such property. The exact cause of lowering cell survival in contact with $\mathrm{C} 4$, that appeared to be not causing significant ATP depletion, is not very clear in our study. It is possible that it caused significant physical damage to the cell membrane during internalization but additional studies are required to confirm such possibility. Based on these evidences, it can be inferred that the common property that caused the cytotoxicity and cellular internalization is the size of the samples. Quite evidently, C2 and C5 possess the smaller size ratios within the sample pool, and C5 bears the minimum size, (in nanometer range) with highest cytotoxicity and cellular internalization. As evident from TEM images, C5 also has acicular 
or sharp sites that may also helped it to penetrate the cellular matter as reported by Li et al. [13] Acicular shape is also present in other samples, like C1 to C3, but that did not help to penetrate the cells. Therefore, this result definitely suggests that sharp edge is not the key factor to consider for cytotoxicity if the size of the particle is not below a certain threshold limit.

\section{Conclusions}

In this report, we have studied and analyzed different aspects and origins of cytotoxicity of diverse varieties of $\mathrm{sp}^{2}$ hybridized carbon materials. Cytotoxicity results using Caco-2 cells confirmed that cell survival rates vary with different types of carbon and nanographene caused the minimal cell survival amongst all the samples studied. The apparent cytotoxicity of non-porous and large carbon particles was attributed to the physical hindrance towards cell growth and that cannot be regarded as a true cause of cytotoxicity. None of carbon samples resulted in reactive oxygen species generation in Caco-2 cells and therefore, it can be concluded that majority of carbon materials do not cause oxidative stress to these cells and hence do not induce oxidation induced malfunction. It was demonstrated that carbon materials do induce ATP depletion in Caco-2 cells in varying proportions and that is the key origin of carbon-induced cytotoxicity. Similar to the cytotoxicity results, nanographene induces the largest ATP depletion resulting in highest cytotoxicity. The visual internalization of nanographene is also confirmed in the TEM images. From the results of this study, it can be concluded that the surface area, pore width, shape and electronic conductivity are not the key contributing factors towards carbon induced cytotoxicity; the size of the particle provides the major role

in it. Acicular shape in combination with small size and associated surface functionality may also provide a competitive cause towards cytotoxicity.

\section{Acknowledgements}

C.H. would like to acknowledge NSF-CBET-1159425 and the Michigan Tech graduate school for funding. We also greatly thank Owen Mills of the Michigan Tech Applied Chemical and 
Morphological Analysis Laboratory for fruitful discussions. TEM (J.C.) experiments were partially conducted at the Center for Nanophase Materials Sciences, which is a DOE Office of Science User Facility. D.S. acknowledges the faculty development award from School of Engineering of Widener University. 
Table 1. Materials characteristics of carbons

\begin{tabular}{|c|c|c|c|c|c|c|}
\hline Species & $\begin{array}{l}\text { Size } \\
(\mu \mathrm{m})\end{array}$ & $\begin{array}{c}\text { Surface area } \\
\left(\mathrm{BET}, \mathrm{m}^{2} / \mathrm{g}\right)\end{array}$ & C (\%) & O (\%) & $\begin{array}{c}\mathbf{s p}^{2} \\
\text { character } \\
(\%)\end{array}$ & $\begin{array}{c}\text { Surface } \\
\text { Functionality } \\
(\%)\end{array}$ \\
\hline $\mathrm{C} 1$ & $100-500$ & 10 & 94.6 & 5.4 & 71.0 & $\begin{array}{r}C-O: 2.4 \\
C=O: 1.2 \\
O-C=O: 1.4 \\
C-O-H: 2.3\end{array}$ \\
\hline $\mathrm{C} 2$ & $5-10$ & 2194 & 92.2 & 7.8 & 73.9 & $\begin{array}{r}C-O: 5.4 \\
C=O: 3.8 \\
O-C=O: 2.3 \\
C-O-H: 3.3\end{array}$ \\
\hline C3 & $20-200$ & 315 & 94.5 & 5.5 & 70.5 & $\begin{array}{r}C-O: 4.3 \\
C=O: 2.3 \\
O-C=O: 1.7 \\
C-O-H: 1.9\end{array}$ \\
\hline $\mathrm{C} 4$ & $20-30$ & 2 & 90.2 & 9.8 & 77.5 & $\begin{array}{r}C-O: 0.0 \\
C=O: 0.0 \\
O-C=O: 0.5 \\
C-O-H: 1.4\end{array}$ \\
\hline $\mathrm{C} 5$ & $0.25-0.05$ & 514 & 86.8 & 13.2 & 20.6 & $\begin{array}{c}C-O: 13.3 \\
C=O: 6.6 \\
O-C=O: 4.8 \\
C-O-H: 1.9\end{array}$ \\
\hline
\end{tabular}


FIGURES

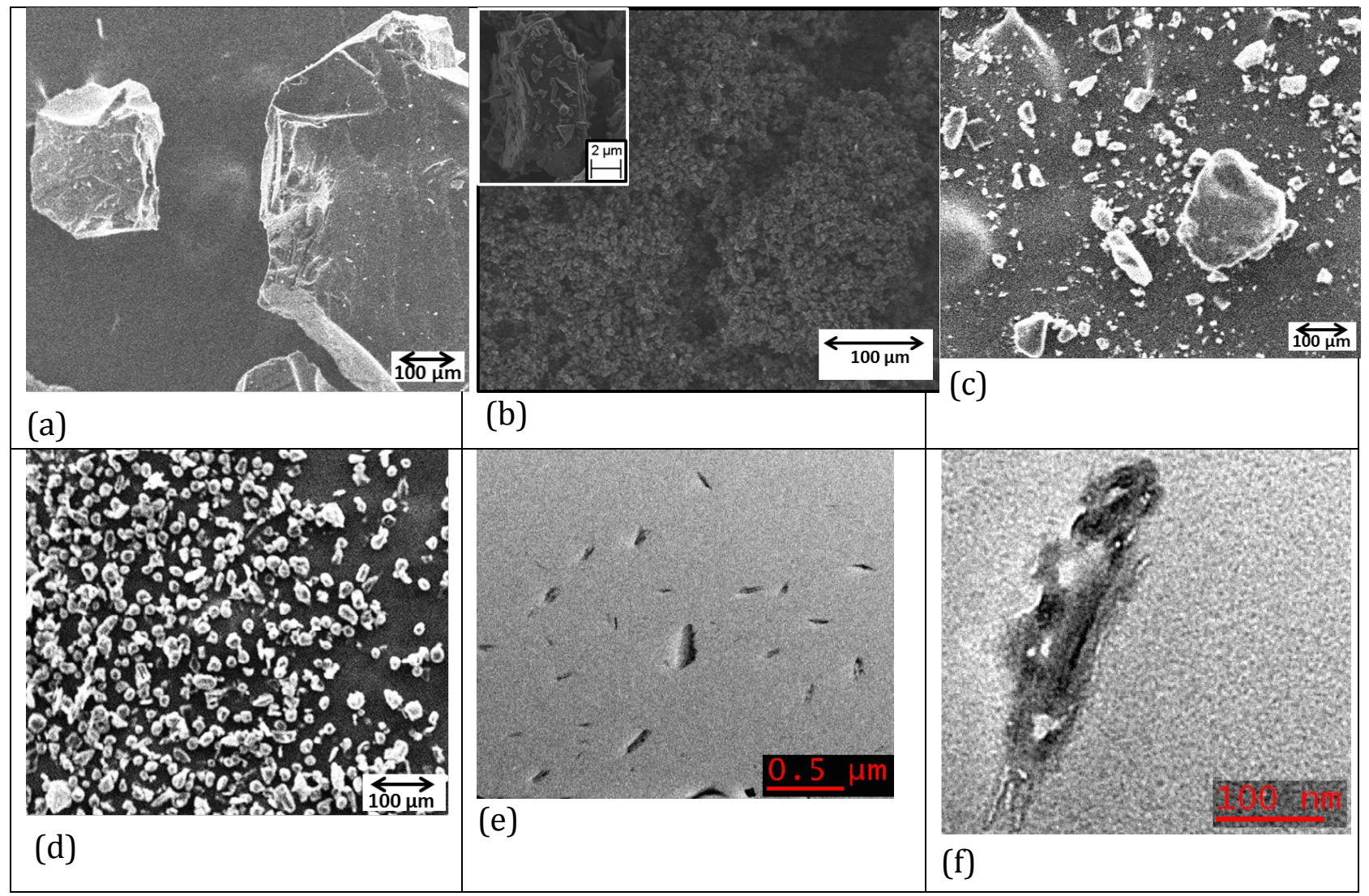

Figure 1. SEM images of C1 (a), C2 (b), C3 (C), C4 (d). TEM images of C5 (e and f) 


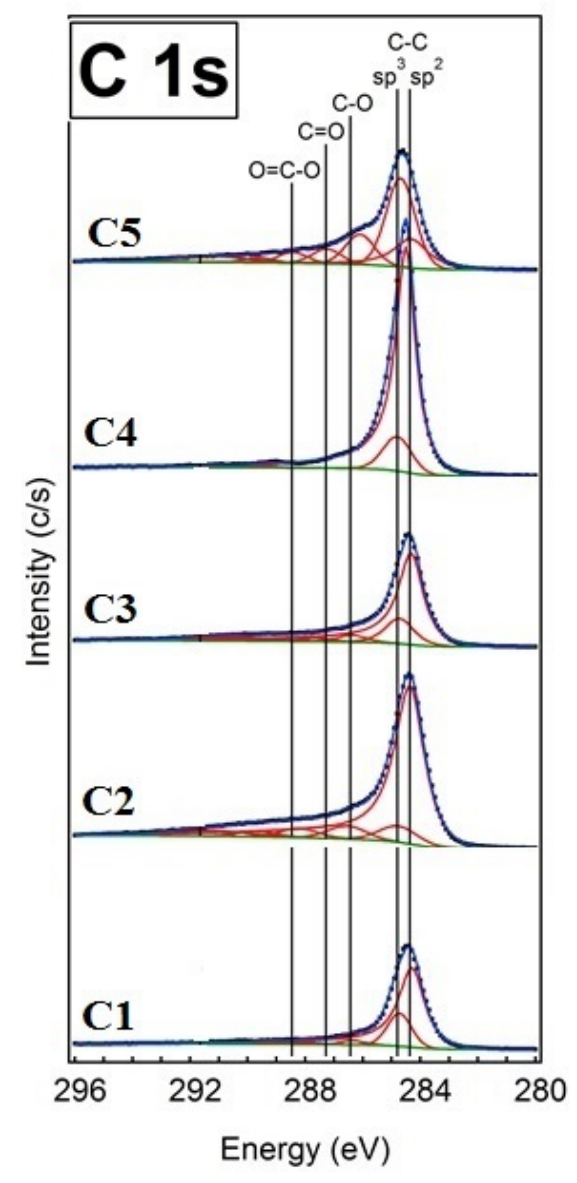

Figure 2. C1s peak fitting of carbon samples from x-ray photoelectron spectroscopy (XPS) results 


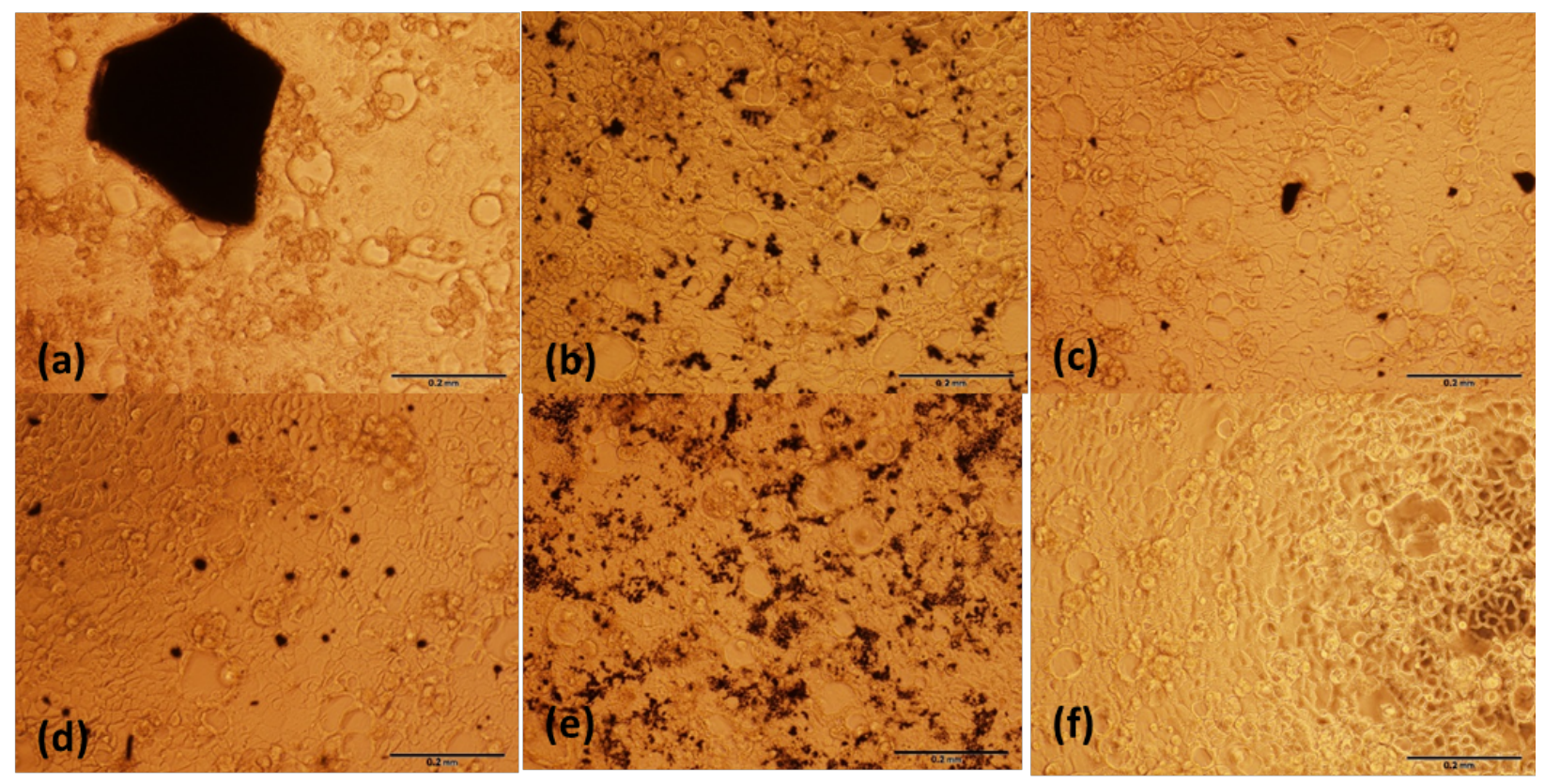

Figure 3. Light microscopic images of the carbon materials in contact with Caco-2 cells after 24 hours of exposure, C1 (a), C2 (b), C3 (c), C4 (d), C5 (e), control cell without carbon (f). The scale bar represents $0.2 \mathrm{~mm}$. 


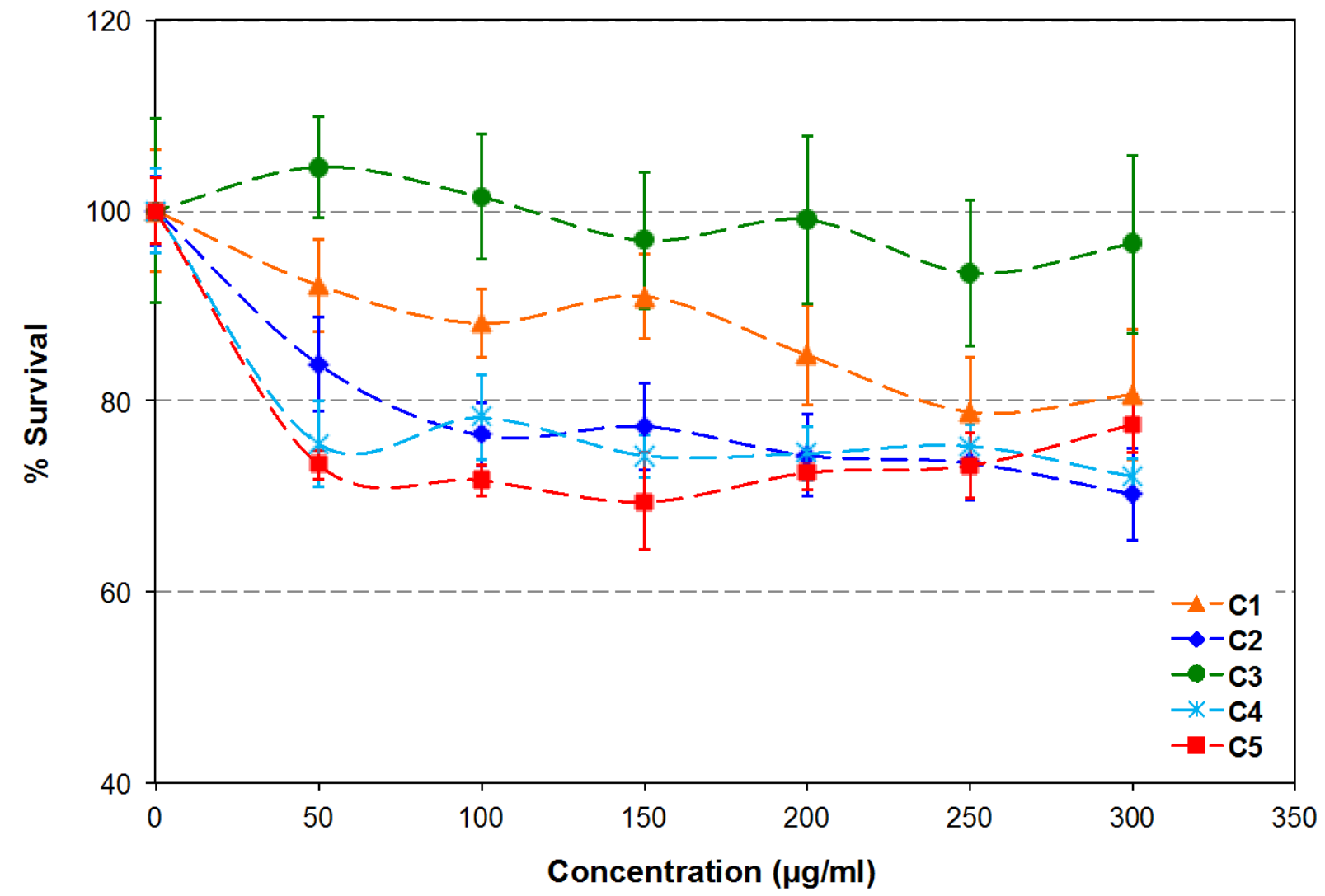

Figure 4. Results of concentration-based cytotoxicity of carbon particles. Cytotoxicity was determined with the MTT cell viability assay. Carbon samples were added to Caco-2 cells and allowed to incubate for 5 days. $100 \%$ viability was assigned to cells that did not contact the carbon samples. 

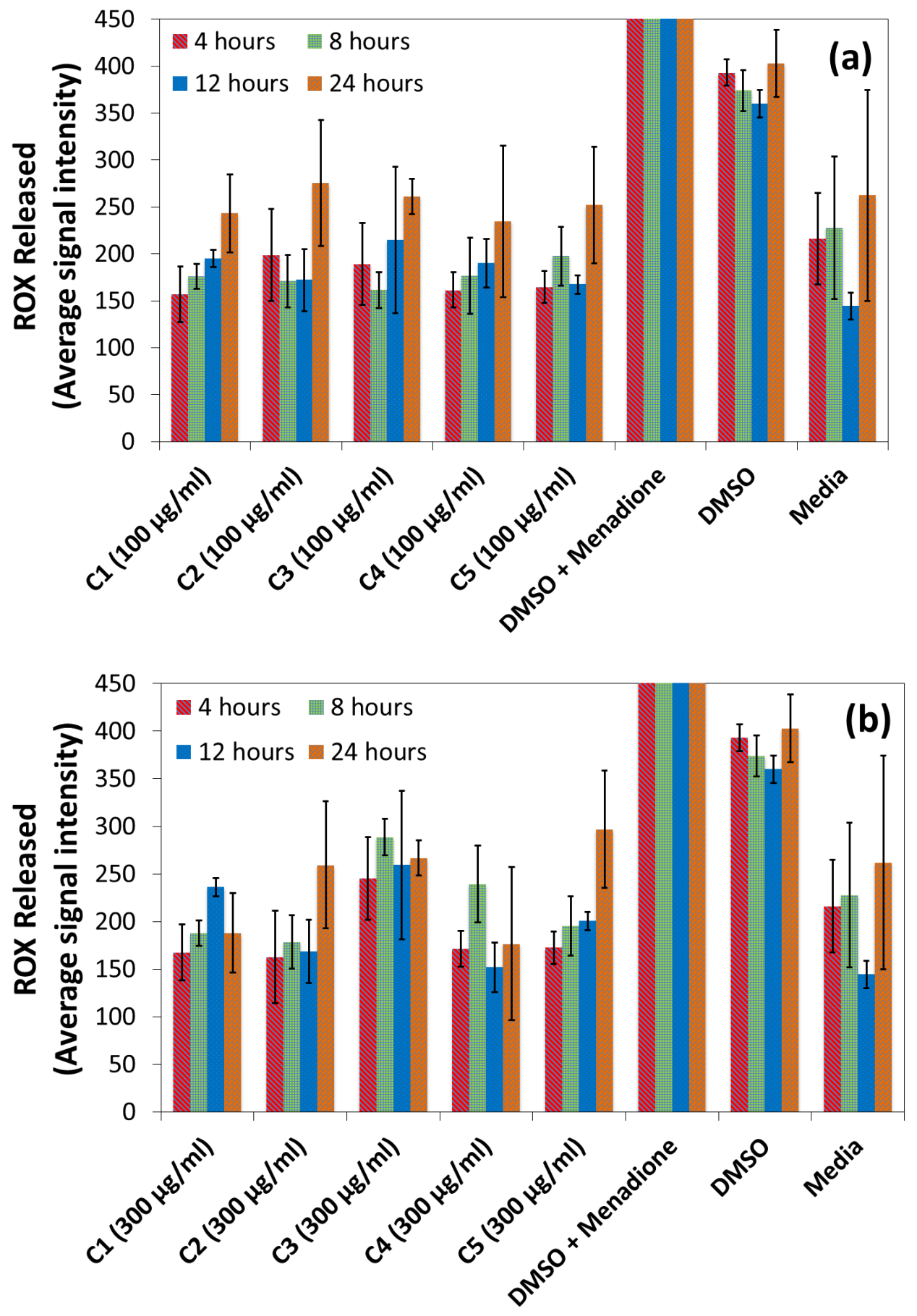

Figure 5. Results of reactive oxygen species (ROS) release study for $100 \mu \mathrm{g}$ (a) and $300 \mu \mathrm{g}$ (b) carbon concentrations. (DMSO+Menadione) is used as positive control and DMSO and Media are used as negative controls. 


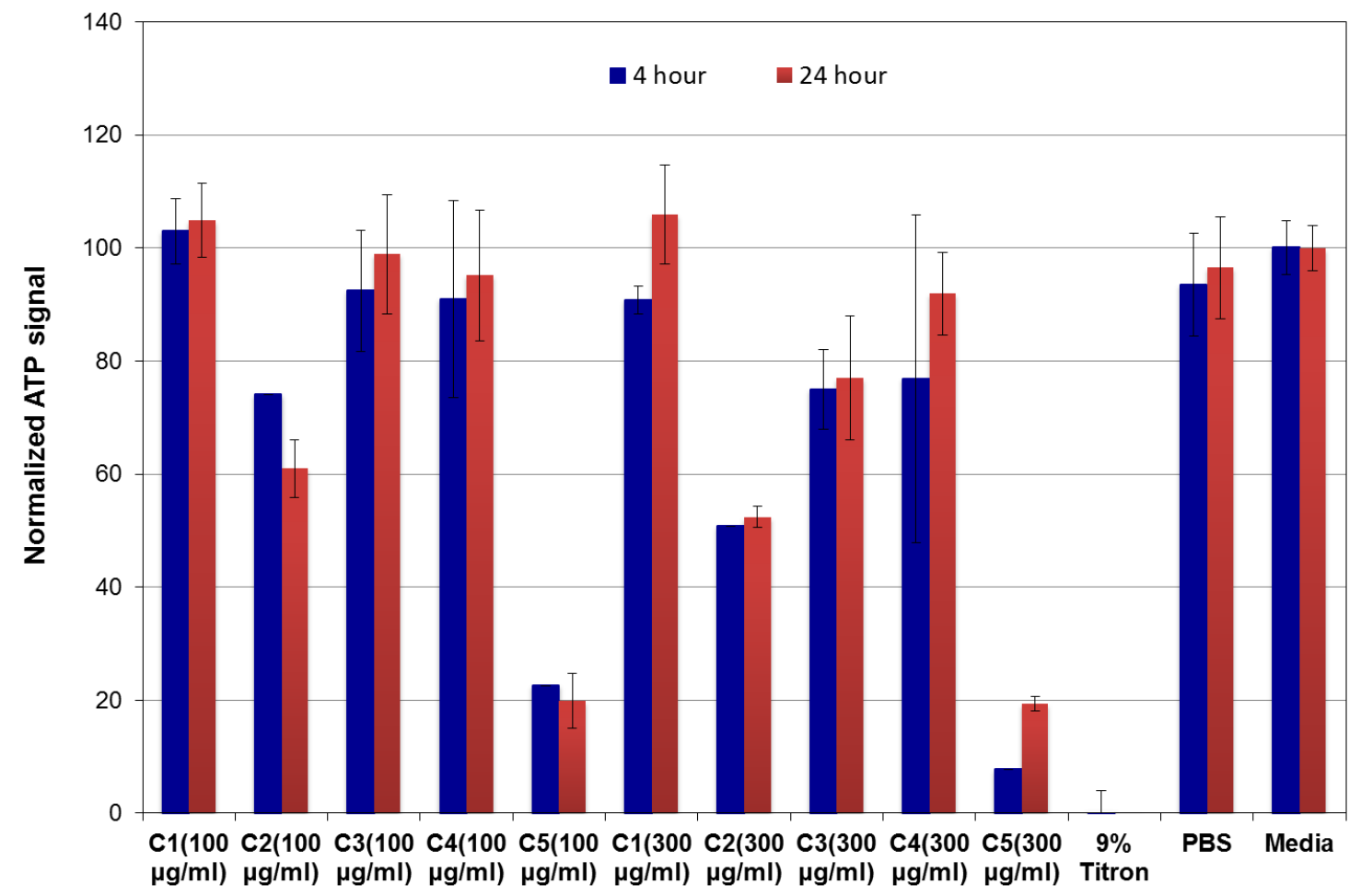

Figure 6. Results of ATP depletion study. Triton is used as negative control and PBS and Media are used as positive controls. 


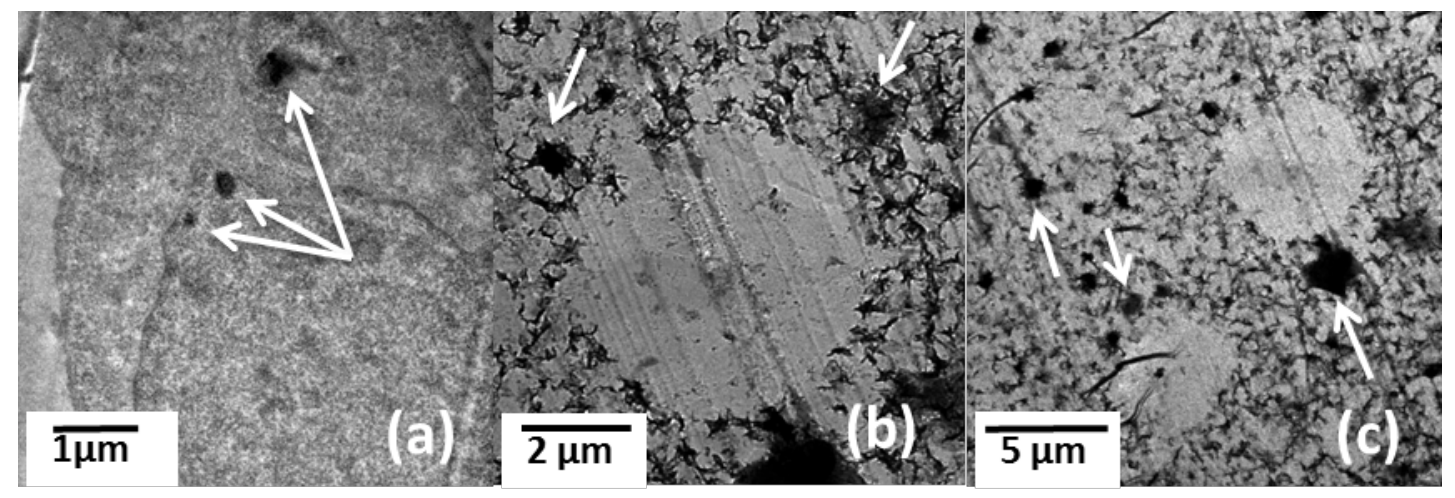

Figure 7. TEM imaging of cellular internalization of C5. The white arrows locate the nanographene particles within the cytoplasmic matter of Caco-2 cell $(a, b, c)$ 


\section{References}

[1] J. Kayat, V. Gajbhiye, R.K. Tekade, N.K. Jain, Pulmonary toxicity of carbon nanotubes: a systematic report, Nanomedicine. 7 (2011) 40-49. doi:10.1016/j.nano.2010.06.008.

[2] D. Saha, E.A. Payzant, A.S. Kumbhar, A.K. Naskar, Sustainable Mesoporous Carbons as Storage and Controlled-Delivery Media for Functional Molecules, ACS Appl. Mater. Interfaces. 5 (2013) 5868-5874. doi:10.1021/am401661f.

[3] A.R. Murray, E. Kisin, S.S. Leonard, S.H. Young, C. Kommineni, V.E. Kagan, et al., Oxidative stress and inflammatory response in dermal toxicity of single-walled carbon nanotubes, Toxicology. 257 (2009) 161-171. doi:10.1016/j.tox.2008.12.023.

[4] J. Muller, F. Huaux, N. Moreau, P. Misson, J.-F. Heilier, M. Delos, et al., Respiratory toxicity of multi-wall carbon nanotubes, Toxicol. Appl. Pharmacol. 207 (2005) 221231. doi:10.1016/j.taap.2005.01.008.

[5] J. Muller, F. Huaux, D. Lison, Respiratory toxicity of carbon nanotubes: How worried should we be?, Carbon. 44 (2006) 1048-1056. doi:10.1016/j.carbon.2005.10.019.

[6] K. Inoue, R. Yanagisawa, E. Koike, M. Nishikawa, H. Takano, Repeated pulmonary exposure to single-walled carbon nanotubes exacerbates allergic inflammation of the airway: Possible role of oxidative stress, Free Radical Biology and Medicine. 48 (2010) 924-934. doi:10.1016/j.freeradbiomed.2010.01.013.

[7] P. Wick, P. Manser, L.K. Limbach, U. Dettlaff-Weglikowska, F. Krumeich, S. Roth, et al., The degree and kind of agglomeration affect carbon nanotube cytotoxicity, Toxicology Letters. 168 (2007) 121-131. doi:10.1016/j.toxlet.2006.08.019.

[8] S.K. Smart, A.I. Cassady, G.Q. Lu, D.J. Martin, The biocompatibility of carbon nanotubes, Carbon. 44 (2006) 1034-1047. doi:10.1016/j.carbon.2005.10.011.

[9] D.B. Warheit, What is currently known about the health risks related to carbon nanotube exposures?, Carbon. 44 (2006) 1064-1069. doi:10.1016/j.carbon.2005.10.013.

[10] R.H. Hurt, M. Monthioux, A. Kane, Toxicology of carbon nanomaterials: Status, trends, and perspectives on the special issue, Carbon. 44 (2006) 1028-1033. doi:10.1016/j.carbon.2005.12.023.

[11] C.M. Sayes, F. Liang, J.L. Hudson, J. Mendez, W. Guo, J.M. Beach, et al., Functionalization density dependence of single-walled carbon nanotubes cytotoxicity in vitro, Toxicology Letters. 161 (2006) 135-142. doi:10.1016/j.toxlet.2005.08.011.

[12] X. Shi, A. von dem Bussche, R.H. Hurt, A.B. Kane, H. Gao, Cell entry of one-dimensional 
nanomaterials occurs by tip recognition and rotation, Nat Nano. 6 (2011) 714-719. doi:10.1038/nnano.2011.151.

[13] Y. Li, H. Yuan, A. von dem Bussche, M. Creighton, R.H. Hurt, A.B. Kane, et al., Graphene microsheets enter cells through spontaneous membrane penetration at edge asperities and corner sites, PNAS. 110 (2013) 12295-12300. doi:10.1073/pnas.1222276110.

[14] M.J. Riding, J. Trevisan, C.J. Hirschmugl, K.C. Jones, K.T. Demple, F.L. Martin. Mechanistic insight into nanotoxicity determined by synchrotron radiation-based Fourier-transform infrared imaging and multivariate analysis. Environment International, 50 (2012) 56-65. doi:10.1016/j.envint.2012.09.009

[15] A. Sasidharan, L.S. Panchakarla, A.R. Sadanandan, A. Ashokan, P. Chandran, C.M. Girish, et al., Hemocompatibility and Macrophage Response of Pristine and Functionalized Graphene, Small. 8 (2012) 1251-1263. doi:10.1002/smll.201102393.

[16] B. Kasemo, Biological surface science, Surface Science. 500 (2002) 656-677. doi:10.1016/S0039-6028(01)01809-X.

[17] E.-J. Chang, H.-H. Kim, J.-E. Huh, I.-A. Kim, J. Seung Ko, C.-P. Chung, et al., Low proliferation and high apoptosis of osteoblastic cells on hydrophobic surface are associated with defective Ras signaling, Experimental Cell Research. 303 (2005) 197206. doi:10.1016/j.yexcr.2004.09.024.

[18] D.G. Corby, W.J. Decker, Management of Acute Poisoning With Activated Charcoal, Pediatrics. 54 (1974) 324-329.

[19] P.A. Chyka, D. Seger, Position statement: single-dose activated charcoal. American Academy of Clinical Toxicology; European Association of Poisons Centres and Clinical Toxicologists, J. Toxicol. Clin. Toxicol. 35 (1997) 721-741.

[20] D. Saha, K.E. Warren, A.K. Naskar, Soft-templated mesoporous carbons as potential materials for oral drug delivery, Carbon. 71 (2014) 47-57. doi:10.1016/j.carbon.2014.01.005.

[21] D. Saha, K.E. Warren, A.K. Naskar, Controlled release of antipyrine from mesoporous carbons, Microporous and Mesoporous Materials. 196 (2014) 327-334. doi:10.1016/j.micromeso.2014.05.024.

[22] D. Saha, T. Moken, J. Chen, D.K. Hensley, K. Delaney, M.A. Hunt, et al., Micro/mesoporous carbons for controlled release of antipyrine and indomethacin, RSC Adv. 5 (2015) 23699-23707. doi:10.1039/C5RA00251F.

[23] X. Wang, P. Liu, Y. Tian, L. Zang, Novel synthesis of Fe-containing mesoporous carbons and their release of ibuprofen, Microporous and Mesoporous Materials. 145 (2011) 
98-103. doi:10.1016/j.micromeso.2011.04.033.

[24] C. Karavasili, E.P. Amanatiadou, L. Sygellou, D.K. Giasafaki, T.A. Steriotis, G.C. Charalambopoulou, et al., Development of new drug delivery system based on ordered mesoporous carbons: characterisation and cytocompatibility studies, J. Mater. Chem. B. 1 (2013) 3167-3174. doi:10.1039/C3TB20304B.

[25] A. Labiano, M. Dai, D. Taylor, W.-S. Young, T.H. Epps III, K. Rege, et al., Slow release kinetics of mitoxantrone from ordered mesoporous carbon films, Microporous and Mesoporous Materials. 160 (2012) 143-150. doi:10.1016/j.micromeso.2012.05.003.

[26] D. Saha, A. Spurri, J. Chen, D.K. Hensley, Controlled release of alendronate from nitrogen-doped mesoporous carbon, Microporous and Mesoporous Materials. 160229 (2016), 8-13. doi:10.1016/j.micromeso.2016.04.014

[27] X. Zhang, T. Zhang, Y. Ye, H. Chen, H. Sun, X. Zhou, et al., Phospholipid-stabilized mesoporous carbon nanospheres as versatile carriers for systemic delivery of amphiphobic SNX-2112 (a Hsp90 inhibitor) with enhanced antitumor effect, European Journal of Pharmaceutics and Biopharmaceutics. 94 (2015) 30-41. doi:10.1016/j.ejpb.2015.04.023.

[28] J. Zhu, L. Liao, X. Bian, J. Kong, P. Yang, B. Liu, pH-controlled delivery of doxorubicin to cancer cells, based on small mesoporous carbon nanospheres, Small. 8 (2012) 27152720. doi:10.1002/smll.201200217.

[29] J. Gu, S. Su, Y. Li, Q. He, J. Shi, Hydrophilic mesoporous carbon nanoparticles as carriers for sustained release of hydrophobic anti-cancer drugs, Chem. Commun. 47 (2011) 2101-2103. doi:10.1039/C0CC04598E.

[30] M.F. Gencoglu, A. Spurri, M. Franko, J. Chen, D.K. Hensley, C.L. Heldt, et al., Biocompatibility of Soft-Templated Mesoporous Carbons, ACS Appl. Mater. Interfaces. 6 (2014) 15068-15077. doi:10.1021/am503076u.

[31] S. Mitragotri, J. Lahann, Physical approaches to biomaterial design, Nat Mater. 8 (2009) 15-23. doi:10.1038/nmat2344.

[32] I. Fenoglio, A. Croce, F. Di Renzo, R. Tiozzo, B. Fubini, Pure-silica zeolites (Porosils) as model solids for the evaluation of the physicochemical features determining silica toxicity to macrophages, Chem. Res. Toxicol. 13 (2000) 489-500.

[33] H.A. Santos, J. Riikonen, J. Salonen, E. Mäkilä, T. Heikkilä, T. Laaksonen, et al., In vitro cytotoxicity of porous silicon microparticles: Effect of the particle concentration, surface chemistry and size, Acta Biomaterialia. 6 (2010) 2721-2731. doi:10.1016/j.actbio.2009.12.043.

[34] F. Delie, W. Rubas, A human colonic cell line sharing similarities with enterocytes as a 
model to examine oral absorption: advantages and limitations of the Caco-2 model, Crit Rev Ther Drug Carrier Syst. 14 (1997) 221-286.

[35] D. Saha, C.I. Contescu, N.C. Gallego, Tetrahydrofuran-Induced K and Li Doping onto Poly(furfuryl alcohol)-Derived Activated Carbon (PFAC): Influence on Microstructure and H2 Sorption Properties, Langmuir. 28 (2012) 5669-5677. doi:10.1021/la3002948.

[36] T. Heikkilä, H.A. Santos, N. Kumar, D.Y. Murzin, J. Salonen, T. Laaksonen, et al., Cytotoxicity study of ordered mesoporous silica MCM-41 and SBA-15 microparticles on Caco-2 cells, European Journal of Pharmaceutics and Biopharmaceutics. 74 (2010) 483-494. doi:10.1016/j.ejpb.2009.12.006.

[37] H.A. Santos, J. Riikonen, J. Salonen, E. Mäkilä, T. Heikkilä, T. Laaksonen, et al., In vitro cytotoxicity of porous silicon microparticles: effect of the particle concentration, surface chemistry and size, Acta Biomater. 6 (2010) 2721-2731. doi:10.1016/j.actbio.2009.12.043.

[38] Z.R. Yue, W. Jiang, L. Wang, S.D. Gardner, C.U. Pittman Jr., Surface characterization of electrochemically oxidized carbon fibers, Carbon. 37 (1999) 1785-1796. doi:10.1016/S0008-6223(99)00047-0.

[39] T. Noda, R. Iwakiri, K. Fujimoto, T.Y. Aw, Induction of mild intracellular redox imbalance inhibits proliferation of CaCo-2 cells, FASEB J. 15 (2001) 2131-2139. doi:10.1096/fj.01-0131com.

[40] B. Zegura, M. Volcic, T.T. Lah, M. Filipic, Different sensitivities of human colon adenocarcinoma (CaCo-2), astrocytoma (IPDDC-A2) and lymphoblastoid (NCNC) cell lines to microcystin-LR induced reactive oxygen species and DNA damage, Toxicon. 52 (2008) 518-525. doi:10.1016/j.toxicon.2008.06.026.

[41]D.M. Brown, I.A. Kinloch, U. Bangert, A.H. Windle, D.M. Walter, G.S. Walker, et al., An in vitro study of the potential of carbon nanotubes and nanofibres to induce inflammatory mediators and frustrated phagocytosis, Carbon. 45 (2007) 1743-1756. doi:10.1016/j.carbon.2007.05.011.

[42] J.F. Turrens, Mitochondrial formation of reactive oxygen species, The Journal of Physiology. 552 (2003) 335-344. doi:10.1111/j.1469-7793.2003.00335.x.

[43] B. Halliwell, Reactive oxygen species in living systems: source, biochemistry, and role in human disease, Am. J. Med. 91 (1991) 14S-22S.

[44] J.A. Imlay, S. Linn, DNA damage and oxygen radical toxicity, Science. 240 (1988) 13021309. 